\title{
Dementia and mobility - issues, needs and solutions
}

\author{
Daniel Bell ${ }^{a}$, Susanne Wolf-Eberl ${ }^{b}$, Patrick Posch ${ }^{b}$ \\ ${ }^{a}$ FACTUM Chaloupka \& Risser OG, Hermann Bahr-Strasse 9/11, 1210 Vienna, Austria \\ ${ }^{b}$ Research \& Data Competence OG, Wiedner Hauptstraße 39/Hofgebäude, 1040 Vienna, Austria
}

ABSTRACT: Structural transition in Austrian society in the larger context of demographic change and growing demands on infrastructure, organization and planning in the public sphere have been discussed profusely in recent years in the context of some projects. While a number of national and international research projects especially focussed on the older age groups and their requirements for staying mobile by developing solutions on the individual, technological and infrastructural level, solutions for people who suffer from cognitive impairment as a result of dementia are still missing. The Austrian research project KOMPETENZ takes the first step in identifying the requirements that people with dementia, their caregivers and other relevant stakeholders in this context have towards public space in order to be able to maintain physical mobility of people affected by dementia. One of the main outcomes of the project included an assessment methodology linking barriers and collective mobility needs to high potential solutions, which supports all relevant stakeholders in the process.

KEYWORDS: Dementia, focus group discussions, expert interviews, social space analysis, maintaining mobility

\section{INTRODUCTION}

Demographic change and its implications for modern transport systems have been at the centre of attention of research from different disciplines and funding programmes in Austria and in other European countries. However, the complex issues of people with dementia in regard to their mobility needs and problems they face have mainly been addressed in context of indoor environments. Proposed innovations in Ambient Assisted Living and concepts of day-care centres have not yet ventured out into public space and mobility systems. Within the Austrian re- search project KOMPETENZ a variety of qualitative research methods were used in order to address all relevant stakeholders in the context of outdoor mobility of people with dementia and also to comprehend how family members, relatives and caregivers affect mobility behaviour of people with dementia. By focussing on existing knowledge on dementia and mobility from previous studies and initiatives and qualitative data collection, the first assessment of the characteristics of the target group in view of mobility needs has been identified. First task was to collect explorative data from a small target group and their caregivers. This rendered a useful insight into indoor and outdoor mobility and provided a link between high potential measures and actual needs of the involved subjects. Since the retreat of people with dementia into privacy might lead to a quicker loss of their physical ability and mobility, another aim was to identify the circumstances which favour and facilitate their participation in public life.

The project's aim was a small-scale exploratory study therefore the empirical data collection required an active participation of the target group which entailed: focus group discussions with people with dementia, their relatives and caregivers, expert interviews with professionals in tangent fields and social space analysis as a part of the qualitative method approach. In addition, concurrent workshops and discussion rounds with an external expert group were established to ensure that the project team focussed on sustainable and feasible solutions and aspects of the research. The combined qualitative efforts not only allowed to establish an active network of stakeholders in the field of dementia and mobility research but also provided a deeper insight into the needs, barriers and necessary solutions concerning issues that have been hardly touched by mobility research before.

The main objective of the project was to collect indicators which can be defined as prerequisites for the safe and comfortable mobility of people with dementia in public space while at the same time the study 
also aimed at a structured depiction of these indicators in form of an assessment methodology.

\section{METHODS}

\subsection{Focus groups discussions with people with dementia and caregivers}

In order to collect input from people with dementia and their relatives and caregivers, a dynamic and adaptable data collection process was established. Focus was on the individual needs of the affected participants. In theory, the basis of the method of focus group discussion is an open and proactive discussion, allowing all participants to actively engage in different topics with the help and support of a trained moderator. However, not all participants or their relatives, who agreed to participate in the study felt comfortable to participate in discussion rounds; their refusal was understandable considering the stigma oftentimes accompanying dementia and Alzheimer's disease. In such cases semi-structured interviews were conducted (via telephone and one face-to-face interview); out of twelve participants two opted for individual interviews.

Focus of the discussion rounds and semi-structured interviews was on mobility problems experienced in everyday mobility habits and on factors which actively or passively influence route choices. The results provided the basis for a social space analysis because they gave new evidence regarding behavioural aspects of outdoor mobility of people with dementia including specific places for sojourning or potential 'black spots' in public space.

Three focus group discussions were conducted. As a rule, usually there are up to fifteen participants in the focus group; however, the sensitivity of the topic led to smaller discussion rounds with maximally four participants. Focus group discussions and semi-structured interviews used the same topics to provide comparable results. Both approaches yielded in-depth insights into the high heterogeneity of the target subjects regarding their living situations and mobility needs.

The recruitment process posed a number of challenges to the research team. The target group of the study were people affected by dementia but who still had the capacity to engage in outdoor mobility without active help of others. To approach them was possible only via gatekeepers from the fields of care giving and medical care. The associated partners in the project however were able to establish the contacts and run the recruitment process. Despite all efforts the group size of a minimum of five participants could not be reached in two discussion rounds. This however had no major impact on both the quality of the collected material or the willingness and active participation of the subjects. Finally, experiences during the discussions and interviews showed that all participants were actively involved and eagerly contributed to the process which secured the success of the project.

\subsection{Expert interviews with medical and mobility experts}

In addition to the focus group discussions and semistructured interviews with persons affected by dementia, experts working in the field of dementia and all dementia related issues were also interviewed in course of expert interviews.

Inclusion of experts (from a given field of expertise) is another popular approach in social sciences as experts can provide professional experience and insights. This method is referred to as semi-standardized approach because it utilizes structured guidelines for questions which are supposed to cover all aspects of the given topic in course of face-to-face interviews (Gogner, A., Littig, B., Menz, W. 2005). Standardized interviews however are generally applied in the context of falsifying hypotheses, to verify assumptions based on empirical evidence. Expert interviews are usually semi-structured and include open questions providing the interviewer with the opportunity to both adapt the interview process to situational dynamics and explore new topics which were not apparent before.

The interviews with experts in the scope of dementia and mobility were based on a structured guideline similar to the face-to-face interviews for people with dementia and their caregivers. This approach aimed at an explorative collection of qualitative data of all aspects of life which affect the everyday mobility of people in different stages of dementia; hence the goal of this process was to generate high-potential solutions to the barriers, difficulties and problems which the target group experiences. Moreover, the more abstract knowledge and expert's contribution to specific issues should provide the research team with indicators for further classification of the target groups and measures how to enable prolonged active participation in public life for people with dementia. 
The active involvement of the group of stakeholders into the research process was meant to exploit those expert views which provide information beyond the individual experiences of the affected persons. The diversity of the research questions and the complexity of the contents demanded a relatively open structure; therefore guidelines were developed in order to support the exploration of unknown aspects of the topic rather than the assessments of known circumstances. While the experts opinions served as an immediate source for the further steps in the research process, they still represent subjective assessments which need to be interpreted and explained in view of the research questions (Mieg, Brunner 2001: 4).

Results of the in-depth literature review and concurrent discussions with project partners (CARITAS, Kuratorium der SeniorInnenhäuser der Stadt Wien, Wiener Mobilitätsagentur) served as useful basis for the structure of the interviews and were also implemented in the final guidelines for interviewers.

The results of the interviews with external experts from the fields of research, mobility and transport and traffic policy provided insight into aspects of dementia and mobility on a societal level and fostered a deeper understanding of the relevance of future measures and policies.

\subsection{Social space analysis and accompanied city walks}

One of the main goals of the KOMPETENZ project was to develop an assessment methodology in order to subsequently involve all relevant criteria for the outdoor mobility of people with dementia in an evaluation process of public space. In the course of assessing the different mobility needs, living situations and individual predispositions which affect mobility, collected data facilitated operationalization of factors which affect public space either positively or negatively regarding mobility related needs for people with dementia. These findings yielded specific knowledge and characteristics of spaces of subjective safety/subjective danger which were incorporated into the subsequent social space analysis.

The social space analysis is an approach which potentially involves a variety of research methods including questionnaires and observations. This process was complemented by participatory observations in selected neighbourhoods and by accompanied walks with people with dementia and included short interviews. Both approaches helped to collect additional

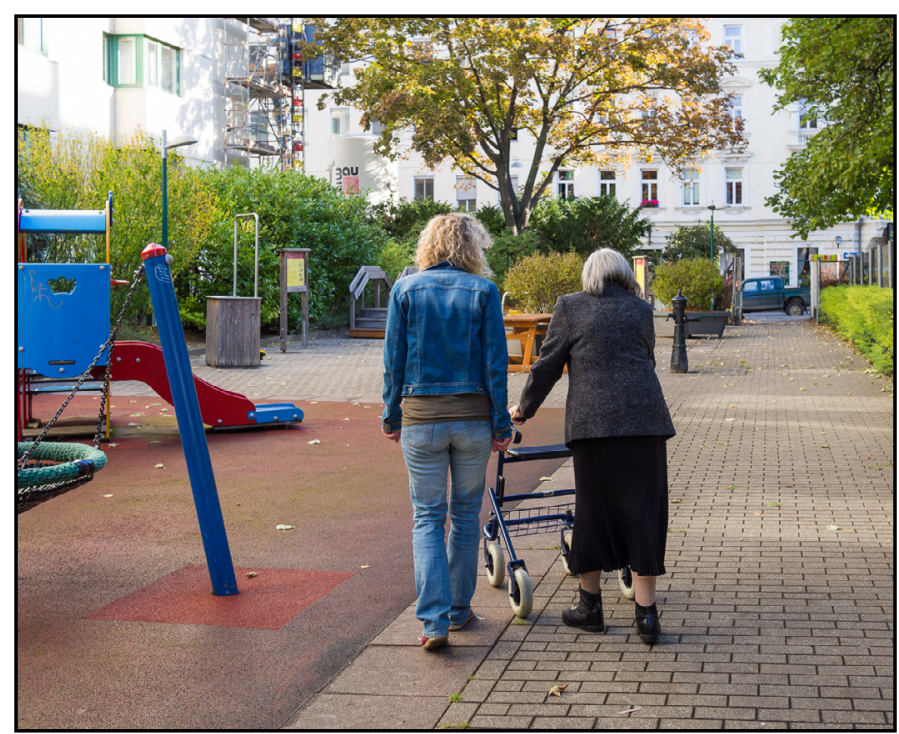

Figure 1: Accompanied walks with people with dementia in urban areas (Copyright 2017 Daniel Bell)

factors which affect the usability and accessibility of public space for people with dementia.

Based on the findings from focus group discussions observation guidelines and areas for investigation were defined. Main focus was on available information and guidance systems, potential barriers, accessibility problems, black spots and best practices based on testimony from the participants. In addition, two accompanied walks with two persons with dementia in the early and medium stages of the disease took place in the vicinity of a day-care centre in Vienna.

The routes of the accompanied walks were defined and chosen by the participants themselves, with only minor suggestions made by the researchers if no immediate conclusion was found. In each project team were two researchers: one researcher in the background with the task to document (photograph, note, etc.) the process and the accompanying researcher accompanying the test person while talking to him/ her. The walking-interview was based on the thinking-out-loud technique where the participant is being encouraged by the researcher to talk about the current experience; this helps to assess of the route characteristics. In addition, both test persons were asked about their general mobility patterns, experiences, changes in mobility behaviour based on their condition, and also for suggestions for improvements and possible solutions for their individual mobility needs.

Both approaches successfully outlined the experiences of people with dementia in public space as they provided empirical input for a better characterization of the factors which directly and indirectly affect accessibility and usability. 


\section{FINDINGS}

Discussions, interviews and observations with people with dementia, their relatives and caregivers provided insight into a specific field of research which has not been fully embraced by the mobility research. When focusing on this target group in course of empirical research studies a variety of issues need to be tackled - regular contact to the research subjects, cooperation with external experts - need to be considered early on. The participatory character of the KOMPETENZ project made it possible to explore in detail conditions under which people with dementia experience public space.

\subsection{General mobility}

One of the main findings of the project underlines the high level of heterogeneity within the group of people with dementia. The differences between examined groups lie not only in the high variety of dementia related diseases, but especially in the differences in the individual lifestyle, in social aspects including living situation and in socio-psychological characteristics. Apart from this, there are still aspects and causalities typical for dementia and its effects on daily life and functional abilities which have not been explained satisfyingly on a medical level.

Our aging population expects a self-determined life which can safeguard a long participation in public life. Aged and sick persons living alone are often socially isolated; while waiting for caregivers, relatives, visitors or meals, they forget about outdoor challenges. This leads to limited contact with the life outside and to the loss of necessary abilities, such as sense of orientation. Caregivers do not have the time or capacity to take their affected relatives for shopping; they cannot accompany them at other activities on a regular basis. The lack of regular physical activity results in a decline of other physical abilities and also cognitive skills. While this especially applies to persons with dementia, it is also highly relevant for elderly persons in general. Physical mobility is necessary for social participation and for mastering the challenges of everyday life. An exclusively indoor care-giving-approach does not provide enough stimulations and challenges. As the disease progresses, people with dementia lose their social skills and their ability to communicate verbally.

It is impossible to make general statements concerning special needs and requirements for barrier- free and comfortable mobility in public space for people with dementia, especially during the early phases of the disease, because those who are affected still live active lifestyles, use public transportation and drive their cars and generally live self-sufficient lives. It is only in these early phases that cognitive decline, loss of memory and orientation, is not the central factor in everyday life yet. Experts agree that in the early stages generalisations are hardly valid. However, especially aggressive forms of Alzheimer's disease can affect functional abilities even early on. The clinical classification of the stages of dementia and their effects on activities of daily living has been discussed only within the limited significance of outdoor mobility and actual participation in public life (Risser et al. 2015). However, more recent analyses of the social, economic and lifestyle related aspects of the associated diseases were referring to impacts of cognitive decline on mobility, although they mainly focus on physical training and the need for additional transport services (Höfler et al. 2015). When people with dementia still live in their own homes, it is the caregivers and relatives who are often in charge of daily planning, including outdoor activities. As long as no incidents happen, the people with dementia are free to move around alone. Unforeseen events and critical incidents can turn into critical turning points; people with dementia may retreat from public life and stop their daily activities, such as shopping, walking around and visiting friends or generally go out. Risk estimation dominates and restricts the mobility pattern and diminishes physical condition and fitness.

As the individual condition is changing and the disease is progressing, the effect on physical condition begins to affect outdoor mobility related needs and attitudes of the person. Especially motor and sensory deficits accompanied by the decline of cognitive capacity can accelerate the retreat from active participation in public life. When people with dementia experience more difficult risks, dangers and comfort levels when venturing outside, a vicious circle of increasing social and activity-related isolation continues. Generally, people with dementia feel safe in known environments. This includes habitual routes and walks in an environment they are familiar with; however this mobility radius and pattern is gradually decreasing. In this situation a small, even a seasonal change in this familiar setting can lead to a loss of orientation and confusion (i.e. construction sites, pavement cafes, detours etc.). During the later 
stages of dementia, when sporadic walks are still possible, finding the way home becomes an increasingly complex and challenging task because recognising buildings, corners or sections of the route becomes increasingly difficult or even impossible especially if something in the setting has changed.

\subsection{Main barriers and mobility problems in public space}

The barriers people with dementia are confronted with again vary greatly based on individual health related preconditions, individual abilities and lifestyles and experiences. Nevertheless, both interviews and social space analysis provided data for structuring the main problems which especially prevent people with dementia form leaving home.

On an individual level increasing levels of disorientation and limited ability to recognize known points of reference and general perception affect autonomous outdoor mobility. These limitations impede the abilities needed for target-oriented actions as planned goals and trip purposes get lost. The consequences of declining cognitive abilities and associated forms of behaviour also involve shame, not only of people with dementia but also of relatives who are usually those who take responsibility for inappropriate behaviour in public and intervene when immediate help is needed. In turn retreat from activities in public due to stigmatization, fear and insecurities can accelerate cognitive decline. The retreat from public space and from public interaction can lead to reductions in physical and social activities which are associated with a more rapid progress of the disease, albeit walking and leisure strolls becoming more and more important with increasing age.

The highest risk associated with people with dementia in public space is related to potentially erratic behaviour or unexpected actions due to disorientation which could both endanger people with dementia themselves or outsiders. As phases of dementia can be fluid it is very hard to forecast actual impacts on mobility. However, interviews with professional caregivers show that dangerous situations which are experienced as a result of mistakes due to cognitive limitations can initiate the next phase of (self-) imposed behavioural restriction. As driving cessation due to decrease of cognitive capacity becomes necessary, the role of public transportation increases as a means for long and medium distance transport for people with dementia. However, learning how to use new forms of transportation, especially for those with limited memory capacity, can become a central barrier. Complex urban transport systems, with varying ticketing schemes, interaction designs for ticketing machines and high passenger volumes during peak hours can make public transport inaccessible. In addition, a high level of audio-visual information can increase cognitive load and lead to disorientation and intensify stress.

The absence of consistent guidance systems in public spaces, including modern signalling systems in complex traffic systems, poses problems - especially for those who are confronted with limited sensory abilities. While the usage of smartphones for way finding- and orientation-tools gains acceptance amongst people with dementia (as it does in the remainder of the target demographic), consistent, easily comprehensible and visible guidance systems at eye level are still necessary and rare. Novel pictograms and signs can be barriers for safe and comfortable navigation and lead to confusion.

Another substantial problem discussed intensely in course of the focus group discussion is the increasing level of automation in modern transport systems and in public space in general. Ticketing machines, cash machines and cash register systems in super markets are often not self-explanatory and lead to confusion. Limited possibilities for human interaction and support in critical situations, where confusion can cause irreversible disorientation, are seen as a main barrier for the target group, especially since interactions on an emotional level are seen as the only way to support somebody who is disoriented and not responsive. In addition, asking other passengers for help or seek support when in need is not always easy due to shame and fear.

Mobility barriers which are not specific to target group but affect it to an even greater extent are related to barrier-free design. As an increasing share of the target demographic is suffering from motor impairments barrier free public spaces and infrastructures are of increasing relevance. People who are dependent on walking aides are especially prone to suffering from uneven surfaces, limited seating and other obstacles in the built environment. This aspect is of course not limited to people with dementia but rather relates to the need for design4all approaches generally called for in planning processes. While these adaptations are generally called for in urban and rural environments, the results of the social space analysis emphasized the need for these meas- 
ures on regional levels where people with dementia a sojourning with a higher frequency (i.e. in the vicinity of day care facilities).

\subsection{Potential measures and solutions}

In general, both medical experts as well as people with dementia themselves and their relatives state that there is a high level of compensation ability in regard to deal with activities which are impacted by cognitive decline. Especially time when to avoid certain situations in traffic (i.e. at peak traffic) and when to leave home for leisure and supply trips are planned well in advance in order to avoid disorientation and stress alike. People with dementia are more likely to forget landmarks and points of interest, which are essential for orientation; hence trips are exercised and planned with all available means (including web-based and smartphone-based trip planning tools).

One of the main measures which is expected to yield benefits on a system and societal level is awareness raising and sensitization of relevant players in order to establish a social safety net for people with dementia if a critical situation occurs in public space. If people with dementia lose orientation or get into a stressful situation due to disorientation, people are needed who can address the situation on an emotional level by avoiding additional stress. Initiatives which help to avoid stigmas and inform about the specific needs of people with dementia should be sensitive in regard to potentially outing those who are affected. Main focus needs to be on establishing a general understanding and knowledge towards the diseases associated with dementia while avoiding stereotypes. Personal assistance in public space is one of the essential factors in this context - this includes learning and training new and complex routes, using public transportation - as customer service employees need to be trained in interactions with the target group. Based on expert opinion case or care-managers, who can inform and train all involved in care giving and facilitate the development of support and mobility strategies to increase safety and comfort for people with dementia in public space.

Walking becomes an increasingly important mode of transport with progressing age, especially among people with dementia who in later stages of dementia start wandering around. While preventive measures for cognitive decline in the course of dementia have not yet been assessed comprehensively on a medically sound level, active mobility and out- door activities are associated with beneficial effects on functional impairments. Supporting measures in the context of walking and outdoor mobility have been assessed especially in the context of innovations which either support way finding or alarm in case of emergency and geo-fencing to support relatives and caregivers in case somebody gets "lost". Specific solutions in this context include navigation aids which are adapted to the actual needs of people suffering from dementia, such as the system by Tervonen et al. (2013), which projects direction arrows right in front of the user based on a worn laser which can also identify potential barriers and dangers. Moreover recent studies not only focussed on providing routing information, but also on collecting movement characteristics and definition of restricted zones to inform caregivers in dangerous situations based on smartphone apps and smartwatches (Schneider et al. 2013). However, these measures are usually only called for in the medium and later phases of dementia where daily routines get mixed up, wandering becomes more common and general activities need to be monitored more closely by those who are taking care. In these phases however modalities of information provision (audio, tactile, or visual) and the specific content provided (arrows, text, etc.) are still being evaluated in order to develop systems which can support people with dementia indoor and outdoor (Sorri et al. 2011). Overall, reduced speeds of motorized traffic and barrier-free infrastructures remain the central factors relevant for subjective safety and comfort when being mobile outdoors for people with dementia.

Spaces for movement and routes in public space are expected to be safe and comfortable for both people with dementia and their relatives when a high level of awareness is present among people working and providing services in these areas. Awareness raising is a complex topic on a societal level however, on regional levels dementia-friendly access points have proven to be working solutions for the provision of information, support and social interaction. It is helpful if passengers or passers-by know where to turn for help if they see a disoriented person or in need of help and assistance. Specific initiatives which can assit people with dementia by providing access- and interaction points in case of disorientation and serve as information point for caregivers and the general public been established on regional levels in Austria in the form of "dementia-pharmacies". These serve as marked institutions with trained personnel who can provide help, inform and connect (Plunger et al. 2017). In 
Austria several initiatives were set up in smaller villages to point attention toward dementia in order to protect people with dementia and also to connect and support caregivers. Caregivers and people with dementia fear about their stigma but accept offers of the initiatives like speeches, validation, singing and creative activities. In Klosterneuburg (a city nearby Vienna, in Lower Austria) an initiative and process started in May 2017 with about 60 participants of different caregiver associations, researchers and locals, stakeholders from Caritas. The common approach included present initiatives like the dementia-pharmacies. After 8 months the initiative obtained full support of the local government for providing dementia services and information (for people with dementia and their relatives and care givers). Local transit services are being provided to many activities.

In the context of way finding and orientation, comprehensible and clearly visible (big, clear and colorcontrasting) traffic signs, guidance systems (i.e. in public transport and public buildings) and landmarks for orientation are important for people with sensory and cognitive impairments. Demands for clear visibility are especially high in problematic lighting situations, readability of building names and text information in public space as these are essential to find unfamiliar points, places or unknown routes.

When using public transportation, ticketing on modern touch interface-based ticket machines, the need to carry additional ID and discount cards can become difficult barriers for people with cognitive impairments. This not only underlines the infrastructural, but also social and service levels in public transportation and public space in general. Awareness raising and training procedures which support public service providers in identifying and supporting people with dementia in public space have already been successfully introduced. In Australia dementia awareness raising campaigns among public bus drivers was initiated and drivers received education and training about how to act and react if somebody loses orientation or cannot remember their destination (NADTC 2015. The Austrian initiative "Mission Dementia" provided police officers with a crash course on how to identify, interact with and support people with dementia, the instruction materials were developed by experts from the field (Auer, 2016).

Increased level of automation and a visible decrease of numbers of customer service employees who can help in case of a problem highlight the need for further developments. Standardization and sim- plifications of the fare structures in public transport and the provision of support personnel would not only increase usability and accessibility for people with dementia of public transport systems. Support, specifically adapted service and identification with new technologies are essential for older people in general and people with dementia in particular to enable them to participate in public life and move about freely (Pelizäus-Hoffmeister, 2015).

An approach to address problems in public space especially discussed in course of the focus groups was an active inclusion of people with dementia into adaptation and planning processes. Active participation via social space analysis for those who are acutely affected by certain limitations in individual mobility highlight their specific needs and the potential problems become apparent in the planning process or evaluation of public infrastructure. Hence an approach to citizen science which specifically involves people with dementia could support the development of sustainable solutions. Best-practice examples can be drawn from projects which aim to encourage and empower the target group members to be vocal about their needs and requirements in public space and to support others who have similar limitations and problems (such as the Mobility Scouts project, which trained older road users to initiate and moderate events to support the development of barrier-free environments) (Andreis et al. 2017: 5)

There has been a controversy during the discussions about semi-public spaces specifically developed for people with dementia - and while there are arguments for developing spaces of retreat where people with dementia can gather and stay and be safe, there are other arguments which highlight the problem of removing public visibility of an increasingly relevant societal problem. This again underlines the high level of heterogeneity of the needs, attitudes and characteristics of the target group and the necessary and useful approaches to support their outdoor mobility.

The general availability of barrier-free and easily accessible spaces in public, where traffic volumes are significantly reduced and free stay and sojourning is encouraged by seating and protection against weather, not only improve comfort of people with dementia but rather meet the needs of the older population as well. While this is not specific to people with dementia, reducing the complexity of everyday mobility, by reducing trip length to essential shopping and medical facilities, will greatly improve outdoor mobility of the older age groups in general. Nevertheless, 
such approaches need to be accompanied by support measures - increasing availability of regional activities which are directly addressing the needs of people with dementia (i.e. transit services for longer trips, rides to events, accompanied walks along selected routes) - which in turn can also take off burdens from relatives and caregivers. If and where such measures are available information must to be provided to affected families.

\section{CONCLUSIONS}

Dementia is often accompanied by an increasing urge to move. As long as affected persons are able to master habitual routes on their own, this urge can be acted out with no restraints. In the course of the disease - with progressing disorientation - the need to move becomes a challenge for caregivers who often cannot and do not want to risk unattended walks of their relatives anymore. The project aimed at providing first insight into the needs of people with dementia and their caregivers from an outdoor mobility perspective based on a small scale exploratory study, hence focus was on gaining access to the target group and developing hypotheses for further research.

When the baby-boom generation reaches higher ages, the numbers of (yet) undetected cases of dementia will rise. Many dementia affected persons will take part in public life and move around in public spaces. Consequently, ensuring a safe and uncomplicated access to public areas and developing guiding aids for persons with dementia are matters of public interest. Defining and designing public training areas where persons with dementia and other memory or cognitive deficits will be able to train and thereby maintain their physical and cognitive abilities also sensitizes the public to the disease and shows how to interact with persons with dementia. Therefore, places for animation and stimulation that focus on maintaining remaining skills rather than being a constant reminder of lost abilities are needed. These places must also be attractive for caregivers.

Prolonging the access to and engagement in public spaces for persons with dementia requires constant efforts by the affected persons themselves and by their relatives and caregivers. It can significantly contribute to a better life quality. Maintaining people with dementia's motor abilities by means of everyday routines is an important condition for maintaining and lengthening his/her participation in public and social life. Basic motor skills need constant exercise, repetition and routines. These are easier achievable outdoors. Moving in public spaces demands the ability of perceiving and processing stimuli on multiple levels. This stimulates the coordination and responsiveness. Physiotherapeutic measures currently take place indoors and in weekly rhythms, because indoor measures are easier workable from the care-taking perspective.

Individual experiences especially on familiar routes, daily trips outdoors are especially relevant in the early stages of dementia where daily activities are still possible. However, even known routes can change temporary or permanently, due to construction works, weather, season, it can be changes in appearance (of buildings) and access (construction sites) or changes of the whole infrastructure that can have a negative and severe impact on orientation and way finding. Here, a social network of passers-by, other passengers and outsiders is essential in order to give support and assistance to a disoriented and visibly lost person without adding more stress and worsening potential traffic conflicts.

As focus groups discussions and expert interviews showed, detailed planned routes are common place for people with dementia who struggle with memory loss and other severe forms of disorientation. Repeated use of specific routes (i.e.: weekly rides to support groups, medical specialist) and the use of pre- and on-trip navigation support - ranging from classic city maps to Google maps on Smartphones -are strategies which provide help and support and facilitate independent mobility in public spaces.

As the decrease of cognitive abilities progresses, accompanying people with dementia gains more importance. Discussions with care giving relatives showed that even in the late stages of dementia outdoor mobility can have a very positive impact on quality of life of those who are affected, even if active participation in social activities is no longer possible. Nevertheless, in these stages walking communities where a small group of people with dementia go on short walks together with an aid could help both, people with dementia and their relatives (the Demenz Initiative Klosterneuburg in Lower Austria currently tries to set up a small walking community).

Some methodological and procedural limitations and existing knowledge need to be discussed in view of further research in this context. One of the limiting factors for the planned participatory approach in the study was the irregular access to the target group 
members. This limitation can be attributed to a variety of factors: firstly, the progression of the disease and other health related contributing factors can rapidly change during the course of a year, making repeated active participation problematic or unfeasible. In addition, actual access to target group of people with dementia who are still self-sufficiently mobile outdoor is challenging due to the fact that these people are usually living at home and only register at self-support groups when cognitive decline has reached a certain level. In this context, access to gate keepers is an essential part of the methodological approach when recruiting samples of the relevant target groups. Nevertheless, the results collected in course of the qualitative research process not only provided great insight into mobility relevant aspects of the daily lives of people with dementia but also highlighted the need for participative processes as these actively include the affected persons in the research.

Already in the early stages of the project, in the course of expert workshops, it became clear that wording is an essential aspect in addressing people with dementia and in developing sustainable solutions. Due to the high level of heterogeneity among people with dementia, factors influencing mobility relevant functional skills vary accordingly. There is a group of people with dementia who do not exhibit mobility behaviour which differs significantly from other representatives from the same demographic group. However, there is also a group at the other end of the spectrum which is not able to stay safely and comfortably mobile in public space due to their state of health.

Acknowledgements: The KOMPETENZ project was funded in the programme "Mobilität der Zukunft" of the Austrian Ministry for Transport, Innovation and Technology (BMVIT) (FFG project number 855011).
Gogner, A., Littig, B., Menz, W. (2005). Das Experten-interview. Theorie, Methode, Anwendung, 2. Auflage, Wiesbaden, Verlag für Sozialwissenschaften.

Höfler, S., Bengough, T., Winkler, P., Griebler, R. (Hg.) (2015): Österreichischer Demenzbericht 2014. Bundesministerium für Gesundheit und Sozialministerium, Wien.

Mieg, H. A., Brunner, B., Mieg, H. A., \& Mieg, H. A. (2001). Experteninterviews: Eine Einführung und Anleitung. Zürich: ETH Zürich, Professur für Mensch-Umwelt-Beziehungen.

Pelizäus-Hoffmeister, H. (2015). Verjüngung des Alters durch Technik. Das subjektive Altersbild im Kontext des technischen Wandels. In: Marcel Plechaty, Frieder R. Lang (Hrsg.), Ältere Menschen in der Mediengesellschaft. Regionale Konferenzen für Bayern. Friedrich-Alexander Universität Erlangen-Nürnberg, S. 82-102. URL: http://www.geronto.fau.de/wp-content/ uploads/2015/06/RegiKon_Dokumentation_2015-06-10.pdf

Plunger, P., Heimerl, K., Tatzer, V., Pichler, B. (2017): Ins Gespräch kommen über Demenz. Toolbox Demenzfreundliche Apotheke. 2. erweiterte und überarbeitete Auflage. Institut für Palliative Care und OrganisationsEthik, IFF, AAU.

Risser, R., Lexell, E.M., Bell, D., Iwarsson, S.. Ståhl. A. (2015). Use of local public transport among people with cognitive impairments - A literature review, Transportation Research Part F 29 (2015) 83-97.

Schneider, C., Willner, V., Henneberger, S. (2013). Elektronische Assistenz für Demenzkranke: Ergebnisse eines ersten Feldtests. (Strobl et al (Hrsg): Angewandte Geoinformatik 2013, Beiträge zum 25. AGIT - Symposium Salzburg, 2013, Wichmann Verlag)

Sorri, L.; Leinonen, E.; Ervasti, M. (2011). Wayfinding Aid for the Elderly with Memory Disturbances. (Proceedings of the 19th European Conference on Information Systems (ECIS 2011), Helsinki, Finland, 9-11 June 2011; p. 137).

Tervonen J., Asghar M.Z., Pulli P., and Yamamoto G. (2013). A navigation aid for people suffering from dementia using a body worm laser device. International Symposium on Medical Information and Communication Technology, ISMICT. 178-182.

\section{REFERENCES}

Andreis, S., Di Mario, A., Mariuzzo, M., Pagano, A. (2017): MOBILITY SCOUTS. Engaging older people in creating an age-friendly environment. Report on research in Italy, Lunaria, Rome Version of 28 February 2017. http://www.mobilityscouts.eu/wp-content/uploads/2017/05/MS_national-report_ Italy.pdf

Auer, S. (2016). Polizeiprojekt „Einsatz Demenz“. Fonds Gesundes Österreich, BVA. http://www.fgoe.org/projektfoerderung/gefoerderte-projekte/FgoeProject_4052/91331.pdf 